\title{
Persistent perceptual grouping effects in the evaluation of simple arithmetic expressions
}

\author{
Jessie Rivera $^{1} \cdot$ Patrick Garrigan $^{1}$
}

Published online: 17 February 2016

(C) Psychonomic Society, Inc. 2016

\begin{abstract}
Landy and Goldstone (2007a, 2010) demonstrated that an explicit rule, operator precedence for simple arithmetic expressions, is enforced in part by perceptual processes like unit formation and attention. When perceptual grouping competes with operator precedence, errors increase. We replicated this result (Exp. 1) and investigated whether perceptual grouping effects persist when the visual stimulus is presented briefly and then masked (Exp. 2) and when verbal recoding is encouraged through vocal expression (Exp. 3). We found that perceptual-grouping effects persisted in the masking condition, suggesting that the mental representations of arithmetic expressions retain visuospatial characteristics. Similarly, verbalization of the expressions did not eliminate perceptualgrouping effects, suggesting that participants were not verbally recoding. In sum, the persistent effects of unit formation and spatial attention emphasize the importance of perceptual processing in the development of human expertise in this domain.
\end{abstract}

Keywords Mental arithmetic · Visual representations

Mental arithmetic involving small numbers and few operations is used in everyday tasks. Quickly and efficiently evaluating simple arithmetic sums and products is also critical for solving more complex problems. Consequently, understanding the mental representations and cognitive operations underlying the evaluation of simple arithmetic expressions is important both in elementary education and for understanding the

Patrick Garrigan

pgarriga@sju.edu

1 Department of Psychology, Saint Joseph's University, 5600 City Ave., Philadelphia, PA 19131, USA mental processing involved in more advanced mathematics, like algebra. There is evidence that the evaluation of simple sums and products (e.g., $2+3$ or $4 \times 5$ ) involves recall mediated by abstract representations of "arithmetic facts" stored in long-term memory (McCloskey, Harley, \& Sokol, 1991). However, the evaluation of expressions involving larger numbers or compound expressions involving more than one operation likely relies on working memory representations (Hitch, 1980) that may be visuospatial, verbal, or both (see, e.g., Baddeley, 1986).

Arithmetic expressions can be encoded as mental representations and solved in this form. Much research suggests that this "mental arithmetic" is supported primarily by verbal working memory. Logie, Gilhooly, and Wynn (1994) found that mental addition involving a series of two-digit numbers, presented through earphones, was impaired by articulatory suppression. They concluded that subvocalization was employed in storing intermediate solutions, presumably in a verbal format. Similar results were obtained with serial, visual presentation. It appears that the phonological loop was involved in accurate calculation, regardless of the modality through which the expression was presented. For three-digit additions presented visually, Fürst and Hitch (2000) found that articulatory suppression impaired performance, provided that presentation was brief (4 s). Consistent with these results, Noël, Désert, Aubrun, and Seron (2001) found that phonological similarity, but not visual similarity, impaired performance in a mental addition task involving three-digit numbers.

Lemaire, Abdi, and Fayol (1996) suggested that arithmetic may come to be supported by the phonological loop because these expressions are trained through oral repetition in elementary education. In their experiments, they showed that for adults verifying simple equations (e.g., $8+4=10$; True? False?), overloading the phonological loop impaired performance. Similarly, Ashcraft, Donley, Halas, and Vakali (1992) 
found that verbal memory load (applied through letter repetition, alphabetization, and word generation) was associated with longer problem-solving latencies even for the verification of simple, visually presented, single-digit additions. Noël, Désert, Aubrun, and Seron (2001) provided converging evidence of the importance of verbal representations for the mental addition of three-digit numbers presented in a visual sequence. Rather than using a concurrent task, in their study, the phonological and visual similarity of the numbers being added was varied. Consistent with the use of a verbal representation, phonological, but not visual, similarity led to confusions.

Interestingly, however, although mental arithmetic appears to involve verbal representations, the evaluation of arithmetic expressions actively available for visual inspection appears to be guided at least in part by visual perception. Landy and Goldstone (2007a, 2010) developed a novel method for probing the nature of the cognitive processing of arithmetic expressions. In their experiments, Gestalt grouping cues (often spacing) were either consistent with the rules of operator precedence or inconsistent with the rules of operator precedence. Operator precedence, or the "order of operations," is a set of rules that dictate the order in which different operations in written arithmetic expressions involving multiple operators of different types are solved. For instance, the expression 2 $\times 5+3$ involves both a multiplication and an addition. By convention, the multiplication is done first.

Consider compound expressions involving one single-digit addition and one single-digit multiplication (e.g., $3+4 \times 5$ ). Expressions with spacing consistent with the rules of operator precedence would have multiplications spaced closer than additions, whereas expressions with spacing inconsistent with the rules of operator precedence would have additions spaced closer than multiplications. Landy and Goldstone found that errors, especially errors in enforcing the rules of operator precedence (operator precedence errors) and errors of symbol identity, were more common when the symbol spacing was inconsistent, for example, $4+3 \times 2$, than when the symbol spacing was consistent, for example, $4+3 \times 2$, with the rules of operator precedence. For expressions like the ones shown above, an operator precedence error would occur if the addition of 4 and 3 were carried out before the multiplication by 2 . Symbol identity errors, on the other hand, occur when an expression is evaluated with the correct operands but at least one incorrect operator-for instance, solving $4+3 \times 2$ as if it were actually $4+3+2$. Landy and Goldstone asserted that the rules of operator precedence are enforced, in part, through low-level visual-perceptual/attentional factors. Similarly, in addition to the shapes of the symbols themselves, the spacing between the symbols influences symbol identification. These spacing effects persist even outside the domain of arithmetic computation: Search response times are faster for multiplication signs than for addition signs (both signs presented in valid expressions), and participants fixate multiplications earlier than additions (Landy, Jones, \& Goldstone 2008).

Symbol spacing, in this case, corresponds to "proximity" in standard Gestalt theory (e.g., Wertheimer, 1923), or a bias to group stimuli that are close to one another. Proximity appears to naturally follow from the contiguous sets of parts that comprise many objects in the world (for a discussion of the ecological validity of proximity as a perceptual grouping cue, see Brunswik \& Kamiya, 1953). Applying proximity to typed symbols may also be quite natural to many readers, given that adjacent letters in words written in, for example, the modern English alphabet are typically more closely spaced than adjacent letters in different words. Although we used proximity exclusively in the experiments presented below, other principles of perceptual organization can also be applied (although perhaps less naturally) to the grouping of symbols in arithmetic expressions (Landy, Allen, \& Zednik, 2014). Though the Gestalt principles are traditionally considered in the context of organization of visual or auditory scenes, even early Gestalt theorists saw the same principles as widely applicable to general problem solving (see, e.g., Ohlsson, 1984). The idea here is somewhat different from Gestalt problem solving, in that traditional perceptual grouping might not reveal a solution, but it could bias the perceiver either toward or away from formal established rules for solving the problem.

Taken together, the results presented above make an interesting prediction. Landy and Goldstone (2007a, 2010) provided evidence that fundamentally visual-perceptual processes help guide the evaluation of visible compound expressions. That is, the pattern of errors they observed was consistent with the idea that the rules of operator precedence are, at least in part, enforced through attentional biases acting on visual representations. Error analysis also suggests that these same biases influence symbol identification. Arithmetic computation on nonvisible expressions - that is, mental arithmetichowever, appears to involve primarily verbal representations. Since the physical spacing of symbols is not explicitly conserved in verbal codes, we expect that the symbol-spacing effects observed by Landy and Goldstone would disappear if the observer could not view the expression throughout its evaluation, but instead had to refer to the mental representation of the expression.

Here we tested whether the effect of symbol spacing (inconsistent or consistent with the rules of operator precedence) persists when arithmetic expressions are solved using mental arithmetic. As we discussed above, there is substantial evidence that mental arithmetic involving visually presented numbers relies on a verbal code. Consequently, one might expect that mentally evaluating an arithmetic expression would not rely on the same visual-perceptual/attentional factors that evaluating a currently visible expressions does. Alternatively, if symbol-spacing effects were observed in 
mental arithmetic, this would imply an interesting interplay between visual and verbal representations in the mental evaluation of arithmetic expressions. Below we replicated the symbol-spacing effect (Exp. 1) and investigated whether symbol-spacing effects persist when the same expressions are mentally evaluated (Exp. 2). Finally, we looked for evidence of symbol-spacing effects when participants were explicitly required to verbalize the expressions (Exp. 3).

\section{Experiment 1}

\section{Method}

Participants A total of 21 undergraduate college students participated in exchange for course credit. For Experiments $1-3$, participants were only included in the analysis if they responded with the correct answer on at least $75 \%$ of all trials. One participant was excluded from the analysis of Experiment 1 for low performance.

Stimuli The stimuli were arithmetic expressions composed of two operators and three operands. The selection of expressions closely followed Landy and Goldstone (2010), Experiment 2. The operators were two additions, two multiplications, an addition followed by a multiplication, or a multiplication followed by an addition $(++, \times x,+\times$, or $\times+)$. The operands were selected from 2,3 , and 4 , with replacement, with the exception of triplets that included only one operand (i.e., 2, 2, 2; 3, 3, 3; and 4, 4, 4). For mixed-operator expressions $(+x$ and $\times+)$, the spacing of the operands was either consistent (closer spacing between operands being multiplied) or inconsistent (closer spacing between operands being added). Here "consistent" and "inconsistent" refer to how perceptual grouping by spacing related to the rules of operator precedence. For expressions with the same operator $(++$ and $\times \times)$, proper implementation of the rules of operator precedence could not be assessed, but the same variation in spacing was used. In sum, all possible combinations ( 4 operand pairs $\times 24$ operator choices $\times 2$ spacings) were each presented on exactly one trial. Trial order was randomized across participants.

Expressions were presented on an iMac computer with a 24 -in. display set at $1,920 \times 1,200$ pixels resolution. Participants were seated approximately $122 \mathrm{~cm}$ from the screen. At this viewing distance, the expressions, presented centered on the screen, subtended approximately $2.7 \times$ $0.4 \mathrm{deg}$ of visual angle. The operand-operator spacing was measured from the center of the addition or multiplication sign to the center of the nearest number. The wide spacing (consistent for additions, inconsistent for multiplications in mixedoperator expressions) was approximately $0.78 \mathrm{deg}$, and the narrow spacing (inconsistent for additions, consistent for multiplications in mixed-operator expressions) was approximately
$0.45 \mathrm{deg}$. For example, in the following expression " $4+3 \times 2$ ", as printed here with consistent spacing, the spacing around the addition sign is wide and the spacing around the multiplication sign is narrow.

Design and procedure For Experiment 1, we employed a within-subjects design in which arithmetic evaluation performance was compared for expressions with consistent and inconsistent spacing. All participants evaluated equal numbers of both types of expressions. The dependent measures included median response times, overall error rates, operator precedence error rates, and symbol identity error rates.

A diagram of the procedure for Experiment 1 is shown in Fig. 1. Participants completed 192 trials, each of which was initiated by a press of the space bar. After the space bar was pressed, a message appeared on the screen indicating the trial number (e.g., "Trial 2 of 192"). After $1.5 \mathrm{~s}$, that message was replaced with one of the arithmetic expressions described above. Participants were to correctly evaluate the expressions as they were presented. They were instructed to compute a correct numeric response and to enter the corresponding digit or digits using the number keys $(0-9)$ on a standard computer keyboard. Upon the first buttonpress participants made to enter their responses, the expression would disappear, and participants could view their response as they completed it. Participants were able to correct mistakes, and once they were satisfied with their response, a press of the space bar would submit it.

Measures For each participant, response times and error rates were measured. The response time for each trial was defined as the elapsed time between the presentation of the expression and the participant's next buttonpress. That is, the response time for each trial corresponded to the time during which the expression (third panel in the sequence shown in Fig. 1) was visible.

Operator precedence and symbol identity error rates were measured separately. Operator precedence errors could only occur on trials when a mixed-operator (one multiplication, one addition) expression was presented. These errors occurred when a participant evaluated an expression as if the rules of operator precedence for multiplication and addition were reversed (in our mixed-operator expressions, adding before multiplying). Symbol identity errors could occur on any trial. These errors occurred when a participant evaluated an expression as if one or both of the operators were the other type (e.g., adding two numbers that should be multiplied).

\section{Results}

Response time and error rate analysis A Wilcoxon signedrank test indicated that errors were more frequent for expressions with inconsistent spacing than for expressions with 


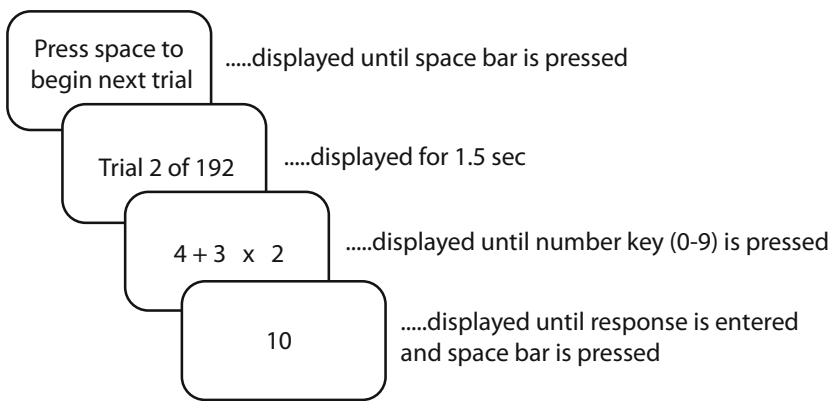

Fig. 1 Diagram of the procedure for a sample trial of Experiment 1

consistent spacing $(z=-2.48, p=.013$, effect size $r=.55)$. Median response times were also longer for expressions with inconsistent spacing than for expressions with consistent spac$\operatorname{ing}\left(M_{\text {con }}=2.57 \mathrm{~s}, S D=0.64 \mathrm{~s} ; M_{\text {incon }}=2.82 \mathrm{~s}, S D=0.74 \mathrm{~s}\right)$, $t(19)=3.51, p=.002, d=0.78$.

More relevant to the present study, however, is that operator precedence error rates were higher for expressions presented with inconsistent spacing than for expressions presented with consistent spacing $(z=-2.87, p=.004, r=.64)$. We did not, however, find a reliable difference in symbol identity error rates between expressions presented with inconsistent spacing and expressions presented with consistent spacing $(z$ $=-0.36, p=.717, r=.08)$. Figure 2, left panel, shows the mean (across participants) proportions of errors of each type, relative to the total numbers of errors made by each participant, for expressions with consistent and inconsistent spacing. Figure 2, right panel, shows the mean (across participants) operator precedence and symbol identity error rate differences between expressions with inconsistent and consistent spacing.

Since Fig. 2, left panel, shows errors by type and condition, each as a proportion of the total error responses for expressions with consistent and inconsistent spacing, it is clear that some errors could not be classified as either operator precedence errors or errors of symbol identity. The participants in Experiment 1 had a mean unclassified error rate of 2.20 errors ( $S D=2.95$ ): $50 \%$ of these errors appeared to be buttonpress errors, in which a single digit, " 1 ," was pressed; $23 \%$ of these errors involved a response in which one of the entered digits was adjacent on the keyboard to the correct key, or was off by one (e.g., entering "12" instead of "13"). These could be either buttonpress or counting errors. The remaining, unclassified errors fit no obvious pattern.

\section{Discussion}

Landy and Goldstone (2010) suggested that the influence of Gestalt grouping effects on the implementation of the rules of operator precedence reflects a deep connection between perception and the learned rules of operator precedence in arithmetic operations. In compound expressions involving both multiplication and addition, like the ones used here,

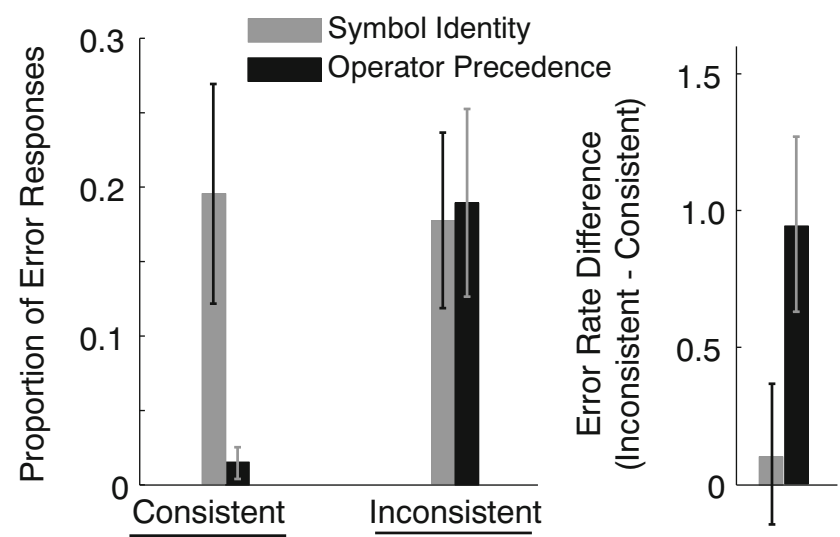

Fig. 2 Experiment 1, error rate analysis. On the left, mean proportions of total errors are shown by error type (symbol identity, operator precedence) and expression spacing (consistent, inconsistent). On the right, mean error rate differences across expression spacings (inconsistent minus consistent) are shown by error type (symbol identity, operator precedence). Error bars are \pm 1 standard error of the mean

multiplications draw attention and form better perceptual groups. Although deliberate application of learned procedures is clearly important (even for expressions presented with inconsistent spacing, errors were relatively rare), the rules of operator precedence do appear to be enforced, in part, through a fundamentally perceptual mechanism. When one considers that inexperienced participants benefit from physical spacing of symbols that is consistent with the rules of operator precedence (Kirshner, 1989), and that both handwritten expressions and typeset algebraic notations employ consistent spacing (Landy \& Goldstone, 2007b), it makes sense that experienced participants might have learned to implicitly utilize this cue for deciding which part of a compound expression should be evaluated first.

Our results are in general agreement with those reported in Experiment 2 of Landy and Goldstone (2010). Operator precedence error rates were higher when spacing was inconsistent. We did not, however, find that symbol identity error rates were reliably different across the inconsistent and consistent expression types. We suspect that the use of spacing as a cue to symbol identity would be more apparent when the processing of other visual cues (e.g., the shapes of the symbols) is compromised (see Exp. 2).

Our results are consistent with studies showing that perceptual grouping and attention, in addition to the deliberate application of learned procedures, guide the evaluation of simple arithmetic expressions. These results, however, are for visible expressions that are continually available for inspection throughout the evaluation procedure. As we discussed above, for mental arithmetic, the literature suggests that verbal, not visual, representations are predominant. Although it is not surprising that the same information might be encoded in different formats depending on task demands (see, e.g., Tversky, 
1969), the use of an exclusively verbal representation would be generally inconsistent with the pattern of errors observed in Experiment 1. For arithmetic expressions, a purely verbal representation would not traditionally preserve information about operator-operand spacing (see the General Discussion, below).

To test this idea, in Experiment 2 we modified the task used in Experiment 1 to require mental arithmetic and to discourage the use of a visual representation. We again compared operator precedence and symbol identity error rates across expression spacing types (consistent vs. inconsistent) to see whether evidence of a visual-perceptual strategy for the evaluation of arithmetic expressions would persist.

\section{Experiment 2}

\section{Method}

Participants A total of 54 undergraduate college students participated in exchange for course credit. Fourteen of these participants were excluded from the analysis for low performance.

Stimuli The same arithmetic expressions presented to participants in Experiment 1 were presented in Experiment 2. In Experiment 2, however, the expressions were visible on the screen for only $500 \mathrm{~ms}$, before they were replaced with a visual mask. Two different visual masks were used. Of the 40 participants included in the analysis, 20 viewed expressions that were masked with white noise (centered on the screen and subtending approximately $6.3 \times 2.4 \mathrm{deg}$ of visual angle), and 20 viewed expressions that were masked with a string of ten digits (randomly selected on each trial, centered on the screen, and subtending approximately $3.9 \times 0.4 \mathrm{deg}$ of visual angle). Both masks covered the part of the screen on which the arithmetic expressions were presented.

Design and procedure The design of Experiment 2 was similar to that of Experiment 1. As in Experiment 1, we employed a within-subjects design in which arithmetic evaluation performance was compared for expressions with consistent and inconsistent spacing. All participants evaluated equal numbers of both types of expressions. The dependent measures included median response times, overall error rates, operator precedence error rates, and symbol identity error rates.

Participants completed 192 trials. Each trial was initiated by the participant, as in Experiment 1. Again, participants were prompted with the trial number and then, $1.5 \mathrm{~s}$ after the participant had initiated the trial, a randomly selected arithmetic expression appeared on the screen for $500 \mathrm{~ms}$ before being replaced by a visual mask (see Fig. 3). This presentation time was chosen in part because it was long enough so that encoding the expressions appeared, from inspection, to be sufficiently easy, but also short enough so that evaluation of the expressions would involve reference to a mental representation. At any time after the mask was presented, participants could begin to input their responses.

Measures The response time for each trial was defined as the elapsed time between the presentation of the expression and the participant's next buttonpress. That is, the response time for each trial corresponded to the time during which the expression and mask (first and second screens in the sequences shown in Fig. 3) were visible.

\section{Results}

Validation of presentation time Performance evaluating consistent expressions in both the white noise and numbers mask conditions of Experiment 2 (93 \% correct, $93 \%$ correct) was similar to performance evaluating the consistent expressions in Experiment 1 (97\% correct). Also, more than $99 \%$ of the response times recorded in Experiment 1 were longer than $1 \mathrm{~s}$, so it appears that a presentation time of $500 \mathrm{~ms}$ was sufficiently short that most trials involved reference to a mental representation of the visually presented expression.

Response time and error rate analysis As in Experiment 1, the error rates were higher for expressions with inconsistent spacing than for expressions with consistent spacing. This was true for both the white noise mask condition $(z=-2.15, p=$ $.031, r=.48)$ and the numbers mask condition $(z=-3.28, p=$ $.001, r=.73)$. Median response times were also longer for expressions with inconsistent spacing than for expressions with consistent spacing for both the white noise mask condition (white noise mask: $M_{\text {con }}=2.48 \mathrm{~s}, S D=0.69 \mathrm{~s} ; M_{\text {incon }}=$ $2.74 \mathrm{~s}, S D=0.53 \mathrm{~s}), t(19)=4.15, p=.001, d=0.93$, and the numbers mask condition (numbers mask: $M_{\text {con }}=2.64 \mathrm{~s}, S D=$ $\left.0.53 \mathrm{~s}, ; M_{\text {incon }}=3.00 \mathrm{~s}, S D=0.62 \mathrm{~s}\right), t(19)=6.60, p<.001$, $d=1.48$.

As in Experiment 1, we again found that operator precedence error rates were higher for expressions presented with inconsistent spacing than for expressions presented with consistent spacing, for both the white noise mask condition $(z=$ $-2.24, p=.025, r=.50)$ and the numbers mask condition $(z=$ $-2.84, p=.004, r=.64)$. However, unlike in Experiment 1, in Experiment 2 we also found that symbol identity error rates were higher for expressions presented with inconsistent spacing than for expressions presented with consistent spacing. This difference was reliable for both the white noise mask condition $(z=-2.55, p=.011, r=.57)$ and the numbers mask condition $(z=-2.42, p=.015, r=.54)$. Figure 4 , left panel, shows the mean (across participants) proportions of errors of each type, relative to the total number of errors made by each participant (within each experiment), for expressions with 
Fig. 3 Expression presentation and masking with a white noise mask (left) and a numbers mask (right) for a sample trial of Experiment 2

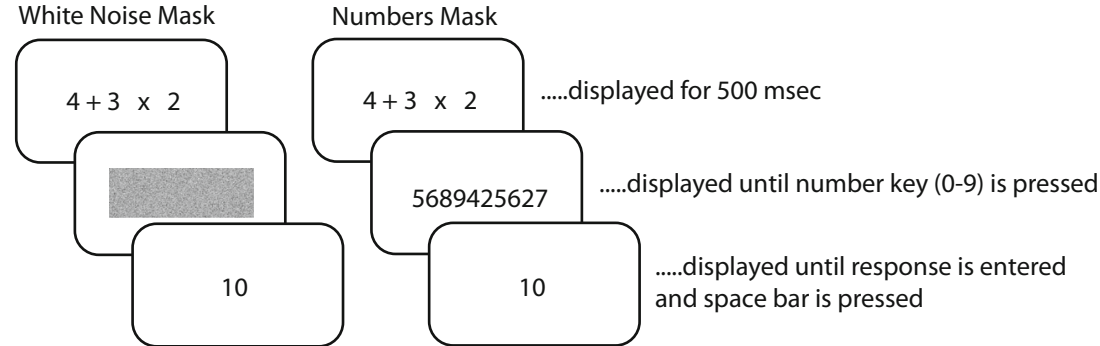

consistent and inconsistent spacing and the noise and numbers masks separately. Figure 4, right panel, shows the mean (across participants) operator precedence and symbol identity error rate differences between expressions with inconsistent and consistent spacing, also separated by mask type.

As in Experiment 1, it is apparent that some errors could not be classified as either operator precedence errors or errors of symbol identity. Participants in the white noise mask condition of Experiment 2 had a mean unclassified error rate of 3.60 errors $(S D=3.15)$. Participants in the numbers mask condition of Experiment 2 had a mean unclassified error rate of 4.95 errors $(S D=2.31)$. Errors in which a single digit, "1," was pressed accounted for $18 \%$ of unclassified errors in the white noise mask condition, and $19 \%$ of unclassified errors in the numbers mask condition. Errors in which one of the digits was entered as the digit on a key adjacent to the correct digit or off by one (e.g., entering " 12 " instead of " 13 ") accounted for $24 \%$ of unclassified errors in the white noise mask condition, and $29 \%$ of unclassified errors in the numbers mask condition. The remaining unclassified errors fit no obvious pattern, but the larger proportion of these errors, relative to Experiment

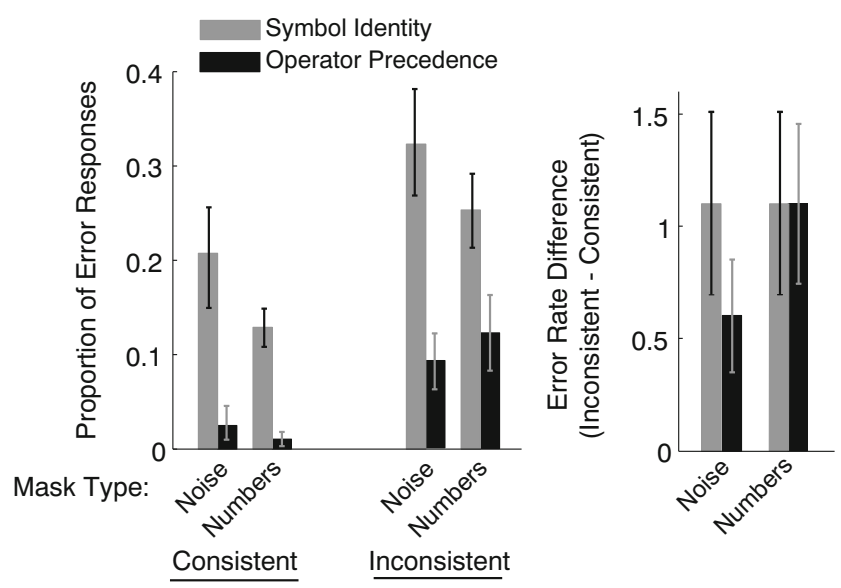

Fig. 4 Experiment 2, error rate analysis. On the left, mean proportions of total errors are shown by error type (symbol identity, operator precedence), mask (white noise, numbers), and expression spacing (consistent, inconsistent). On the right, mean error rate differences across expression spacings (inconsistent minus consistent) are shown by error type (symbol identity, operator precedence) and mask (white noise, numbers). Error bars are \pm 1 standard error of the mean
1, may have been due to increased encoding errors as a result of the visual masking.

\section{Discussion}

Experiment 2 was designed to require participants to evaluate visually presented arithmetic expressions using a mental representation. Visual masking (white noise, numbers) was employed to further challenge the use of a stored visual representation. A white noise mask carries no semantic information and, by definition, has equal power in all spatial frequencies. The numbers mask was also used, to test whether or not a masking stimulus composed of readable symbols and a more closely matched power spectrum would be more effective in disrupting visual processing. We expected that operator precedence errors would occur independent of operator-operand spacing if a mental representation were used, because earlier studies had indicated that mental arithmetic operates on verbal representations in which symbol spacing information is lost. Furthermore, we expected that forming a verbal representation would be a natural strategy if visual masking were used to compromise the formation of a visual representation (see, e.g., Breitmeyer \& Öğmen, 2006).

The median response times for evaluating the expressions (as estimated by the time between presentation of the expression and the first keypress for inputting the numeric response, averaged across participants) were similar in Experiment 1 ( $M$ $=2.96 \mathrm{~s}, S D=0.77 \mathrm{~s})$ and Experiment $2(M=2.88 \mathrm{~s}, S D=$ $0.63 \mathrm{~s}$ ). Since the presentation time (prior to masking) was restricted to $500 \mathrm{~ms}$, it is reasonable to assume that participants relied on a mental representation of the expression once it was no longer visible.

We used two different types of mask: white noise and random numbers. In both cases, we found a reliable difference in operator precedence error rates between the consistent and inconsistent spacing conditions. This result indicates that these expressions were evaluated using a representation that retained visuospatial information; that is, verbal recoding did not occur. This was surprising, given the earlier studies, reviewed above, showing that mental arithmetic is mediated by verbal representations. It is important to note, however, that our results do not exclude the possibility of any role for a verbal code. Instead, they show that the representation that 
supports the mental evaluation of simple arithmetic expressions maintains visuospatial characteristics - that is, a representation that is at least in some sense visual and nonverbal. In the General Discussion, we consider how a hybrid representation of this sort might be used.

Besides these earlier results, though, we had other reasons to expect to find verbal recoding of the arithmetic expressions presented in Experiment 2. Verbal recoding is a wellestablished strategy for maintaining information in working memory. Even 2-year-old children are capable of boosting memory performance through strategic verbal recoding (Kibbe \& Feigenson, 2014). Human memory has been shown to flexibly adapt the format in which information is stored in response to task demands (e.g., Tversky, 1969). By changing the task demands so that visual processing was curtailed, we expected that the expressions would be recoded in a verbal format in which symbol spacing information would be lost. This was not the case.

One alternative explanation for our results is that, perhaps some participants evaluated the expressions very quickly, using the visually available information. If this occurred, we would expect participants who made operator precedence errors when evaluating expressions with inconsistent spacing to also have faster response times. We investigated this alternative hypothesis, by comparing the response times for participants in Experiment 2 who exhibited more operator precedence errors for expressions with inconsistent spacing to those for all other participants. This alternative hypothesis - that excess operator precedence errors for expressions with inconsistent spacing should be associated with faster response times-was not supported. The median response times for participants in Experiment 2 who exhibited more operator precedence errors for expressions with inconsistent spacing $(M=2.86 \mathrm{~s}, S D=0.64 \mathrm{~s})$ were very similar to the median response times for all other participants $(2.89 \mathrm{~s}, S D=0.64 \mathrm{~s})$.

One could still be concerned that the visual encoding and evaluation of expressions using a visual representation might always happen very quickly, with response times being dominated by other processes like response initiation. If this were the case, longer response times would not be associated with increased error rates. More generally, perhaps the masking did not sufficiently disrupt visual processing of the stimulus to the extent that a verbal recoding strategy was warranted. We suspect that this was not the case. Visual masking appeared to make the evaluation of expressions considerably more challenging, as was indicated by the differential participant exclusion rates in Experiments 1 and 2. In Experiment 2, five (white noise condition) and nine (numbers mask condition) participants were excluded from the analysis due to failure to correctly evaluate $75 \%$ of the expressions. In Experiment 1, the same performance threshold only resulted in the exclusion of one participant. If the encoding and evaluation of expressions occurred rapidly while the expressions were still visible, we would expect the masking to have little impact on task difficulty.

Unlike in Experiment 1, in Experiment 2, we also found a reliable difference in symbol identity error rates between the consistent and inconsistent spacing conditions. Recall that symbol identity errors are errors in which an operator in an expression (e.g., a "+") is inappropriately processed as a different operator (e.g., a " $\times$ "), so that, for example, the expression " $4+3 \times 2$ " would be erroneously evaluated as " $4 \times 3 \times$ 2 ". The fact that symbol identity errors were more common in the evaluation of expressions presented with inconsistent spacing suggests that the spacing between symbols in an expression is used as a cue to symbol identity, at least under conditions in which the identification of symbols is challenging.

Increased symbol identity errors under conditions of compromised visual encoding may be related to "cue recruitment," in which an uninformative visual cue (e.g., presentation position on a computer screen) can be trained in order to disambiguate a bistable figure (e.g., a Necker cube; Qi, Saunders, Stone, \& Backus, 2006). In our case, spacing, a formally uninformative cue to symbol identity, was being used as if it were predictive. For this learning to occur, observers must have been exposed to scenarios in which relatively narrow spacing was associated with multiplication and relatively wide spacing was associated with addition. As Landy and Goldstone (2010) discussed, this relationship does appear in some natural contexts.

In Experiment 2, we created conditions under which participants needed to use mental arithmetic to evaluate simple arithmetic expressions. We also used visual masking to impair the formation of a visual representation of the expressions. Still, we found no evidence of verbal recoding. In Experiment 3, we again looked for evidence of verbal recoding. Here, however, instead of inhibiting formation of a visual representation, we promoted the formation of a verbal representation.

\section{Experiment 3}

\section{Method}

Participants A total of 21 undergraduate college students participated in exchange for course credit. One participant was excluded from the analysis for low performance.

Stimuli The same arithmetic expressions presented to the participants in Experiments 1 and 2 were presented in Experiment 3.

Design and procedure The design of Experiment 3 was similar to the designs of Experiments 1 and 2. Again, we 
employed a within-subjects design in which arithmetic evaluation performance was compared for expressions with consistent and inconsistent spacing. All participants evaluated equal numbers of both types of expressions, and the dependent measures included overall error rates, operator precedence error rates, and symbol identity error rates.

Participants completed 192 trials. Each trial was initiated by the participant, as in Experiments 1 and 2.

Participants were instructed that, upon presentation of the expression, they should immediately verbalize the expression, out loud, and press the space bar as soon as they had completed their verbalization. The expression disappeared when the space bar was pressed, at which point participants would complete the trial by entering their response in the same manner as in Experiments 1 and 2 (see Fig. 5). The verbalization of expressions was intended to promote the formation of a verbal representation of each expression.

\section{Results}

Error rate analysis As in Experiments 1 and 2, error rates were higher for expressions with inconsistent spacing than for expressions with consistent spacing $(z=-2.31, p=.021, r=$ $.52)$. The primary results of interest in Experiment 3 closely matched those found in Experiment 1. Operator precedence error rates were higher for expressions presented with inconsistent spacing than for expressions presented with consistent spacing $(z=-2.55, p=.011, r=.57)$. As in Experiment 1, we did not find a reliable difference in symbol identity error rates between the expressions presented with inconsistent and consistent spacing $(z=-1.66, p=.096, r=.37)$. Figure 6 , left panel, shows the mean (across participants) proportions of errors of each type, relative to the total number of errors made by each participant, for expressions with consistent and inconsistent spacing. Figure 6, right panel, shows the mean (across participants) operator precedence and symbol identity error rate differences between expressions with inconsistent and consistent spacing.

As in Experiments 1 and 2, it is apparent that some errors in Experiment 3 could not be classified as either operator precedence errors or errors of symbol identity. The participants in Experiment 3 had a mean unclassified error rate of 1.80 errors $(S D=1.36)$. Errors in which a single digit, "1," was pressed accounted for

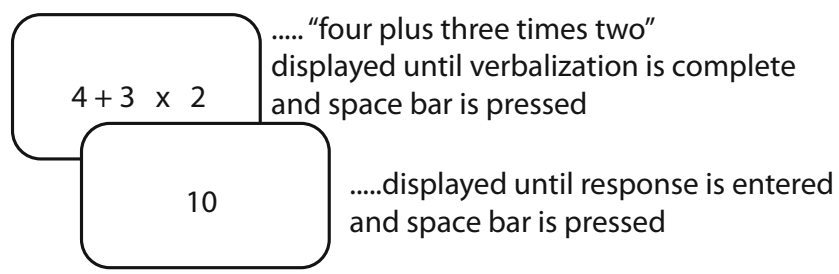

Fig. 5 Expression presentation and articulation procedure for a sample trial of Experiment 3

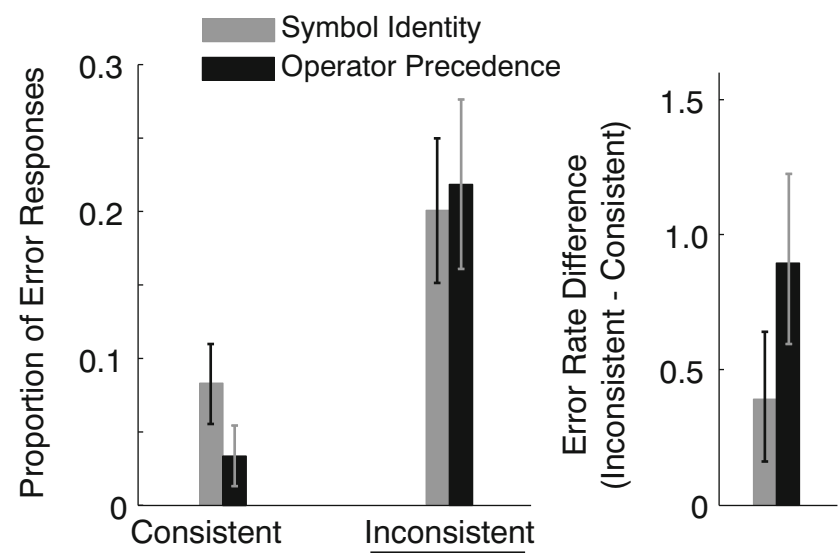

Fig. 6 Experiment 3, error rate analysis. On the left, mean proportions of total errors are shown by error type (symbol identity, operator precedence) and expression spacing (consistent, inconsistent). On the right, mean error rate differences across expression spacings (inconsistent minus consistent) are shown by error type (symbol identity, operator precedence). Error bars are \pm 1 standard error of the mean

$22 \%$ of the unclassified errors. Errors in which one of the digits was entered as the digit on a key adjacent to the correct digit or off by one (e.g., entering " 12 " instead of "13") accounted for $28 \%$ of the unclassified errors. The remaining unclassified errors fit no obvious pattern.

\section{Discussion}

In Experiment 2, we hypothesized that evaluating arithmetic expressions using a visual representation would be less advantageous when the presentation time was restricted and masking was used to disrupt visual processing. Still we found no evidence of verbal recoding in Experiment 2. In Experiment 3, we took a different tack.

Here, we attempted to encourage participants to recode the visually presented expressions in a verbal format. The method was adapted from Experiment 2 of Brandimonte, Hitch, and Bishop (1992), in which the authors showed that the presentation of verbal labels near images led to verbal recoding. In our Experiment 3 , participants were required to articulate the expression, after which the expression disappeared and they could enter their response. In this way, verbal recoding might be quite natural, perhaps automatic, or at least was more apparent as a strategy.

Articulating the expression would also, presumably, present the stimulus in a format more closely matched to the format of verbal working memory, and so the processing costs of recoding the expression would, to some extent, be mitigated. Finally, forming a verbal code presumably requires the time necessary to articulate it. Since verbalizing the expression was required in Experiment 3, any temporal costs would also be mitigated. 
We still, however, found evidence of the use of a visual representation: Operator precedence errors were reliably more frequent in the inconsistent spacing condition. Symbol identity errors, on the other hand, were not reliably more frequent in the inconsistent than in the consistent spacing condition. This fits with the results and interpretation of Experiments 1 and 2. When visual processing was not interrupted by masking (Exp. 1), the identifying visual information of the symbols themselves dominates the less reliable identifying information of the spacing between the symbols. When masking was employed (Exp. 2), symbol spacing played a greater role in symbol identification, leading to more symbol identity errors in the evaluation of expressions with inconsistent spacing. In Experiment 3, expressions were again presented for an extended period of time and without masking. Just as in Experiment 1, the visual information of the symbols themselves dominated the information of the spacing between the symbols, and again we found no reliable evidence that symbol identity error rates depended on whether or not the spacing was consistent with the rules of operator precedence.

\section{General discussion}

Some people report strong visual associations to simple computations, and even to individual numbers (Seron, Pesenti, Noël, Deloche, \& Cornet, 1992). Neurophysiological data tell a similar story for arithmetic. In college students, arithmetic computation involves brain regions associated with mental imagery and visual working memory (Zago et al., 2001). Brain imaging of a mental calculation "prodigy" yielded similar results (Pesenti et al., 2001).

On the other hand, considerable behavioral research, reviewed above, supports the view that simple arithmetic is mediated, perhaps primarily, by verbal representations. Other studies have suggested a more nuanced perspective. For example, the roles of verbal and visual representations may be related to the mathematical operation performed (Imbo, Vandierendonck, \& Vergauwe, 2007; Lee \& Kang, 2002), dependent on an individual's problem-solving skills (Rasmussen \& Bisanz, 2005), and even affected by whether the expression is presented in horizontal or vertical format (Trbovich \& LeFevre 2003). A study of Chinese undergraduates (Xinlin \& Qi, 2003) showed that the time to solve onedigit additions depended on whether Arabic or Chinese numbers (large visual difference, no auditory difference) were used. No effect was found for one-digit multiplications, however. The researchers concluded that the students had used a visual representation for additions but an auditory representation for multiplications (Xinlin \& Qi, 2003). Besides indicating that the verbal or visuospatial dichotomy may be overly simplistic, these results also cast doubt on the idea of a single, amodal representation for arithmetic expressions.
Perhaps visual representations are more important in arithmetic than some of the verbal-recoding evidence suggests. Using an entirely different approach, the methodology adopted here, Landy and Goldstone (2007a, 2010) showed that formally irrelevant visuospatial characteristics of visually presented arithmetic expressions can affect performance. But do these perceptual-grouping effects persist in the domain of mental arithmetic? This is the question we addressed here.

If mental arithmetic operated on exclusively verbal representations, then spacing effects should disappear when arithmetic expressions are mentally evaluated. We found, however, that operand-operator spacing influences arithmetic expression evaluation even when evaluation of the expression happens at least partly after the visually presented stimulus is no longer available for inspection. In sum, our results suggest that recoding of the visual stimulus into either a purely verbal or an abstract, amodal format does not occur, even when visual masking might discourage the use of a visual representation, or when explicit verbalization of the expression is required. It appears that the use of a visual representation is a persistent feature of the evaluation of visually presented arithmetic expressions.

This result lends support to the idea that formal rules, like operator precedence, are realized in part through perceptual grouping and attentional strategies. Besides the evidence that mental arithmetic involves verbal representations, verbalization is also a common strategy used by participants in memory experiments. This is why investigations of visual working memory often employ articulatory suppression to ensure that participants are not recoding a visual stimulus as a verbal description. We expected, therefore, that perceptual grouping effects would disappear in Experiments 2 and 3. Our finding that perceptual strategies are employed in mental arithmetic supports the idea that the offloading of cognitive rules onto perceptual mechanisms is, in fact, quite fundamental.

Finding no evidence of verbal recoding was also surprising because these stimuli were, in many ways, ideal for verbal recoding. There were relatively few symbols and operators, all with predefined, easy-to-articulate names. Verbal overshadowing (Carmichael, Hogan, \& Walter, 1932; Schooler \& Engstler-Schooler, 1990) was not a concern. Verbal recoding seemed a natural strategy in Experiment 2, and might perhaps be unavoidable in Experiment 3.

These expectations also fit with research on dual-code representations of objects. Johnson, Paivio, and Clark (1989) suggested that representations initiated in one modality can activate verbal representations if the object is identified as familiar. Representations of unfamiliar objects may also be subject to verbal recoding if a verbal description can be generated (Lacey \& Campbell, 2006). Furthermore, verbal recoding appears to disrupt the formation of mental images that support spatial reasoning (Brandimonte, Hitch, \& Bishop, 1992). In our case, we expected that verbal recoding of 
familiar symbols (numbers, operators) would disrupt the formation of mental images that might preserve veridical spacing among the symbols. Our results, however, suggest otherwise.

It is possible, of course, that a verbal code could preserve spacing information, perhaps as pauses in subvocalizations. This does, however, blur the behavioral distinction between verbal and visual encoding in the present context. It also requires a relatively sophisticated mapping from the visual stimulus to the verbal code, since perceptual-grouping effects like those reported here can be induced with grouping cues not typically found in written or typeset arithmetic expressions (Landy \& Goldstone, 2007a). On the other hand, there is evidence that, in some cases, a great deal of spatial information is preserved well in representations derived from language (Zwaan \& Radvansky, 1998), so even if a verbal code were to replace a visuospatial code, it could still give rise to a representation that retained spacing information. For example, Avraamides, Klatzky, Loomis, and Golledge (2004) had participants form mental images from verbal descriptions of scenes, and found that representation of the spacing among objects in verbally relayed mental images was functionally equivalent to similar representations being generated from actual viewing.

How can we reconcile the present results with the studies reviewed above that suggest that mental arithmetic operates on verbal representations? The answer may be that mental evaluation of arithmetic expressions involves a complicated interplay among information encoded in a variety of formats: verbal, visual, and amodal. For example, planning a procedure for evaluating an expression may involve a visual representation in which simple features like symbol shapes, element spacing, and parentheses guide attention and form perceptual groups. Subparts of the expression are then sequentially read and encoded as a verbal representation. Generating solutions to subparts, like simple products and sums, may involve the recall of arithmetic facts that are neither verbal nor visual.

Understanding how information encoded in different formats is integrated in a single process remains a significant challenge. However, there may be good reasons to believe that this added complexity is justified by the benefits of utilizing both visual and verbal working memory at once. Whereas we have evidence that verbal and visual working memory share resources (e.g., Mate, Allen, \& Baqués, 2012; Salmela, Moisala, \& Alho, 2014), information that could be stored in either a visual or a verbal format might be more efficiently stored in one or the other. Perhaps for the expressions used, the advantages of processing a pictorial, rather than a verbal, representation justify maintaining a visual format even under the less-than-ideal conditions of Experiments 2 and 3. One might also imagine that the evaluation of arithmetic expressions taxes verbal working memory, so that visual working memory is required to maintain adequate performance. Sorting these issues out presents an interesting avenue for future research.

\section{Symbol identity errors}

Participants use the relative spacing around operators as a cue to operator identity (Landy \& Goldstone, 2010). The results of our Experiment 2 are consistent with this interpretation. We did not, however, find that symbol identity errors were more common when spacing was inconsistent with the rules of operator precedence in Experiments 1 and 3. The critical difference between Experiment 2 (both masking conditions) and Experiments 1 and 3 appears to be that the visual encoding of expressions was substantially more challenging in Experiment 2.

We cannot, of course, draw conclusions from null results, but the pattern across experiments suggests implicit integration of spacing information as a cue to symbol identity. In Experiment 2, short presentation times and masking impaired visual encoding, so that the reliability of the typically dominant visual cues (symbol shapes) was lower. Under these conditions, an optimal cue combination strategy might begin to use other sources of information, like spacing, to inform symbol identity. Cue combination rules of this sort have been widely observed in perceptual phenomena (see, e.g., Young, Landy, \& Maloney, 1993).

\section{Why does spacing convey information?}

In many computer programming languages, "whitespace" is ignored by the compiler. Still, programmers use whitespace to make their code easier to read and understand. Besides making code appear less cluttered, whitespace can also impart a perceptual organization that makes logical groupings more apparent.

With similar logic, Landy, Allen, and Zednik (2014) argued that symbol systems that use perceptual attributes to convey information are efficient. Dropping the multiplication sign in algebraic notation (e.g., $3 y+4)$ implies that multiplication precedes addition. Shared biases, such as that "closer together" implies "consider together," make some designs more intuitive than others. This idea is central to the principle of pictorial realism (Roscoe, 1968), which posits that a welldesigned display's physical characteristics (e.g., the upward motion of a dial) should be compatible with the user's mental representation of the value the display is intended to convey (e.g., an increasing quantity).

\section{Arithmetic, perceptual learning, and instruction}

Traditional education often emphasizes the learning of facts and beliefs that can be readily put into words (Bereiter \& Scardamalia, 1996). Experts, however, often have access to important relations they cannot articulate. This is partly how a novice chess player who can state all the rules of chess differs from an expert who might be unable to verbalize the strategies 
she employs. This learning of relations is one form of "perceptual learning" (Kellman \& Garrigan, 2009).

Perceptual learning refers to experience-induced changes in the way that information is extracted from the environment (Gibson, 1969). Much perceptual-learning research has focused on simple judgments or discriminations, like determining whether two line segments are collinear (Wertheimer, 1975). However, perceptual learning also influences complicated and novel problem solving. Knoblich, Ohlsson, Haider, and Rhenius (1999) showed that puzzle-solving performance was impaired when the solution involved "chunk decomposition" (Knoblich, Ohlsson, \& Raney, 2001) of a stronger, rather than a weaker, perceptual grouping. For even more complicated problems, like geometric proofs, considering alternative representations of a problem (i.e., different perceptual organizations of the same geometric diagram) may be critical for discovery of the solution (Ohlsson, 1990).

From the results presented here, one might conclude that using perceptual grouping to guide operator precedence or inform symbol identification should be avoided. After all, the three experiments presented here demonstrated the use of perceptual strategies through their association with increased error rates. However, the use of perceptual strategies in cases in which they may, superficially at least, seem inappropriate may reflect a more general principle in the development of human expertise. Evaluating arithmetic expressions may initially involve learning explicit rules for manipulating symbols, but experience allows for some of these rules to be offloaded onto low-level perceptual mechanisms, like visual attention. The ability to use basic neural machinery in novel ways may be of critical importance. As Goldstone, Landy, and Son (2010) discussed, evolutionarily speaking, we have only recently begun evaluating arithmetic expressions. Our ability to coopt perceptual machinery that has served humanity well for millennia demonstrates the remarkably efficient adaptability of the human brain.

More practically, it is important for instructors to take into account the implications of the fact that, like it or not, learners employ perceptual strategies. Perceptual learning is usually not the focus of formal instruction, but it may be necessary for achieving high levels of performance across many domains. Perceptual learning allows experts to free up cognitive resources so that more complex, higher-order relations can be considered. But perceptual learning not only distinguishes novices from experts, it also distinguishes the novice from the slightly more experienced. Two children might both be able to recall and explain the order of operations equally well. However, a child who can evaluate arithmetic expressions without explicitly recalling the order of operations may have cognitive resources available for deeper learning.

Author note The authors express thanks to David Landy and Robert Goldstone for helpful suggestions on this work.

\section{References}

Ashcraft, M. H., Donley, R. D., Halas, M. A., \& Vakali, M. (1992). Working memory, automaticity, and problem difficulty. In J. I. D. Campbell (Ed.), The nature and origins of mathematical skills (pp. 301-329). Amsterdam: Elsevier.

Avraamides, M. N., Klatzky, R. L., Loomis, J. M., \& Golledge, R. G. (2004). Use of cognitive versus perceptual heading during imagined locomotion depends on the response mode. Psychological Science, 15, 403-408. doi:10.1111/j.0956-7976.2004.00692.x

Baddeley, A. (1986). Working memory. New York: Oxford University Press, Clarendon Press.

Bereiter, C., \& Scardamalia, M. (1996). Rethinking learning. In D. R. Olson \& N. Torrance (Eds.), The Handbook of education and human development: New models of learning, teaching and schooling (pp. 485-513). Cambridge: Blackwell.

Brandimonte, M. A., Hitch, G. J., \& Bishop, D. V. M. (1992). Verbal recoding of visual stimuli impairs mental image transformations. Memory \& Cognition, 20, 449-455. doi:10.3758/BF03210929

Breitmeyer, B. G., \& Öğmen, H. (2006). Visual masking: Time slices through conscious and unconscious vision (2nd ed.). Oxford: Oxford University Press.

Brunswik, E., \& Kamiya, J. (1953). Ecological cue-validity of "proximity" and of other Gestalt factors. American Journal of Psychology, 66, 20-32.

Carmichael, L., Hogan, H. P., \& Walter, A. A. (1932). An experimental study of the effect of language on the reproduction of visually perceived forms. Journal of Experimental Psychology, 15, 73-86.

Fürst, A. J., \& Hitch, G. J. (2000). Separate roles for executive and phonological components of working memory in mental arithmetic. Memory \& Cognition, 28, 774-782. doi:10.3758/BF03198412

Gibson, J. J. (1969). Principles of perceptual learning and development. New York: Prentice-Hall.

Goldstone, R. L., Landy, D. H., \& Son, J. Y. (2010). The education of perception. Topics in Cognitive Science, 2, 265-284.

Hitch, G. J. (1980). Developing the concept of working memory. In G. Claxton (Ed.), Cognitive psychology: New directions (pp. 154-196). London: Routledge and Kegan Paul.

Imbo, I., Vandierendonck, A., \& Vergauwe, E. (2007). The role of working memory in carrying and borrowing. Psychological Research, 71, 467-483. doi:10.1007/s00426-006-0044-8

Johnson, C. J., Paivio, A. U., \& Clark, J. M. (1989). Spatial and verbal abilities in children's crossmodal recognition: A dual coding approach. Canadian Journal of Psychology, 43, 397-412. doi:10. 1037/h0084229

Kellman, P. J., \& Garrigan, P. (2009). Perceptual learning and human expertise. Physics of Life Reviews, 6, 53-84.

Kibbe, M. M., \& Feigenson, L. (2014). Developmental origins of recoding and decoding in memory. Cognitive Psychology, 75, 5579.

Kirshner, D. (1989). The visual syntax of algebra. Journal for Research in Mathematics Education, 20, 274-287. doi:10.2307/749516

Knoblich, G., Ohlsson, S., Haider, H., \& Rhenius, D. (1999). Constraint relaxation and chunk decomposition in insight problem solving. Journal of Experimental Psychology. Learning, Memory, and Cognition, 25, 1534-1555. doi:10.1037/0278-7393.25.6.1534

Knoblich, G., Ohlsson, S., \& Raney, G. E. (2001). An eye movement study of insight problem solving. Memory \& Cognition, 29, 1000 1009.

Lacey, S., \& Campbell, C. (2006). Mental representation in visual/haptic cross-modal memory: Evidence form interference effects. Quarterly Journal of Experimental Psychology, 59, 361-376.

Landy, D., Allen, C., \& Zednik, C. (2014). A perceptual account of symbolic reasoning. Frontiers in Psychology, 5, 275. doi:10.3389/ fpsyg.2014.00275 
Landy, D., \& Goldstone, R. L. (2007a). Formal notations are diagrams: Evidence from a production task. Memory \& Cognition, 35, 2033 2040.

Landy, D., \& Goldstone, R. L. (2007b). How abstract is symbolic thought? Journal of Experimental Psychology. Learning, Memory, and Cognition, 33, 720-733. doi:10.1037/0278-7393.33.4.720

Landy, D., \& Goldstone, R. L. (2010). Proximity and precedence in arithmetic. Quarterly Journal of Experimental Psychology, 63, 1953-1968. doi:10.1080/17470211003787619

Landy, D., Jones, M. N., \& Goldstone, R. L. (2008). How the appearance of an operator affects its mathematical precedence. In B. C. Love, K. McRae, \& V. M. Sloutsky (Eds.), Proceedings of the 30th Annual Conference of the Cognitive Science Society (pp. 2109-2114). Austin: Cognitive Science Society.

Lee, K., \& Kang, S. (2002). Arithmetic operation and working memory: Differential suppression in dual tasks. Cognition, 83, B63-B68.

Lemaire, P., Abdi, H., \& Fayol, M. (1996). The role of working memory resources in simple cognitive arithmetic. European Journal of Cognitive Psychology, 8, 73-103. doi:10.1080/095414496383211

Logie, R. H., Gilhooly, K. J., \& Wynn, V. (1994). Counting on working memory in arithmetic problem solving. Memory \& Cognition, 22, 395-410.

Mate, J., Allen, R. J., \& Baqués, J. (2012). What you say matters: Exploring visual-verbal interactions in visual working memory. Quarterly Journal of Experimental Psychology, 65, 395-400.

McCloskey, M., Harley, W., \& Sokol, S. M. (1991). Models of arithmetic fact retrieval: An evaluation in light of findings from normal and brain-damaged subjects. Journal of Experimental Psychology. Learning, Memory, and Cognition, 17, 377-397. doi:10.1037/ 0278-7393.17.2.377

Noël, M., Désert, M., Aubrun, A., \& Seron, X. (2001). Involvement of short-term memory in complex mental calculation. Memory \& Cognition, 29, $34-42$.

Ohlsson, S. (1984). Restructuring revisited: I. Summary and critique of the Gestalt theory of problem solving. Scandinavian Journal of Psychology, 25, 65-78.

Ohlsson, S. (1990). The mechanism of restructuring in geometry. In Proceedings of the Twelfth Annual Conference of the Cognitive Science Society (pp. 237-244). Hillsdale: Erlbaum.

Pesenti, M., Zago, L., Crivello, F., Mellet, E., Samson, D., Duroux, B., . . . Tzourio-Mazoyer, N. (2001). Mental calculation in a prodigy is sustained by right prefrontal and medial temporal areas. Nature Neuroscience, 4, 103-107.

Qi, H., Saunders, J. A., Stone, R. W., \& Backus, B. T. (2006). Demonstration of cue recruitment: Change in visual appearance by means of Pavlovian conditioning. Proceedings of the National Academy of Sciences, 103, 483-488. doi:10.1073/pnas.0506728103

Rasmussen, C., \& Bisanz, J. (2005). Representation and working memory in early arithmetic. Journal of Experimental Child Psychology, 91, 137-157.

Roscoe, S. N. (1968). Airborne displays for flight and navigation. Human Factors, 10, 321-322.

Salmela, V. R., Moisala, M., \& Alho, K. (2014). Working memory resources are shared across sensory modalities. Attention, Perception, \& Psychophysics, 76, 1962-1974. doi:10.3758/s13414-014-0714-3

Schooler, J. W., \& Engstler-Schooler, T. Y. (1990). Verbal overshadowing of visual memories: Some things are better left unsaid. Cognitive Psychology, 22, 36-71. doi:10.1016/0010-0285(90)90003-M

Seron, X., Pesenti, M., Noël, M. P., Deloche, G., \& Cornet, J. A. (1992). Images of numbers, or "When 98 is upper left and 6 sky blue.". Cognition, 44, 159-196.

Trbovich, P. L., \& LeFevre, J.-A. (2003). Phonological and visual working memory in mental addition. Memory \& Cognition, 31, 738-745. doi:10.3758/BF03196112

Tversky, B. (1969). Pictorial and verbal encoding in a short-term memory task. Perception \& Psychophysics, 6, 225-233.

Wertheimer, G. (1975). Visual acuity and hyperacuity. Investigative Ophthalmology, 14, 570-572.

Wertheimer, M. (1923). Untersuchungen zur Lehre von der Gestalt: II. Psychologische Forschung, 4, 301-350. [Condensed in Selection 5 in W. D. Ellis (Ed.). (1938). A source book of Gestalt psychology. New York, NY: Harcourt, Brace.]

Xinlin, Z., \& Qi, D. (2003). Representation formats for addition and multiplication facts. Acta Psychologica Sinica, 35, 345-351.

Young, M. J., Landy, M. S., \& Maloney, L. T. (1993). A perturbation analysis of depth perception from combinations of texture and motion cues. Vision Research, 33, 2685-2696.

Zago, L., Pesenti, M., Mellet, E., Crivello, F., Mazoyer, B., \& TzourioMazoyer, N. (2001). Neural correlates of simple and complex mental calculation. Neurolmage, 13, 314-327.

Zwaan, R. A., \& Radvansky, G. A. (1998). Situation models in language comprehension and memory. Psychological Bulletin, 123, 162-185. doi:10.1037/0033-2909.123.2.162 